\title{
Design of Interactive Medical Devices: Feedback and Its Improvement
}

\author{
Yunqiu Li, Patrick Oladimeji \\ Carlos Monroy, Abigail Cauchi, Harold Thimbleby \\ Computer Science Department \\ Swansea University, Swansea, Wales, UK, SA2 8PP \\ \{Yunqiu.Li, P.Oladimeji, C.Monroy, A.Cauchi\}@ swansea.ac.uk \\ H.Thimbleby@swansea.ac.uk
}

\author{
Dominic Furniss, Chris Vincent \\ Ann Blandford \\ UCLIC, University College London \\ Gower Street, London, UK, WC1E 6BT \\ \{D.Furniss, C.Vincent, A.Blandford\}@ucl.ac.uk
}

\begin{abstract}
The design of medical devices directly affects the way healthcare practitioners carry out their daily tasks. Users welcome design that takes into account the clinical environment, in which the device is operated and is compatible with their workflow. However, if the design fails to fit, the likelihood of errors increases, which will put patient safety at risk. In this paper, we report current practice related to UCD (User Centred Design) in the context of medical device, focusing on end user feedback mechanisms deployed in pre-market and post-market phases of the device lifecycle. The results of an interview and workshop study are reported, revealing shortcomings in current feedback channels. In reaction to these shortcomings, we discuss the advantages and feasibility of enabling automatic feedback channel in medical device design, to ensure the quality and the effectiveness of feedback.
\end{abstract}

\section{INTRODUCTION}

Amongst various interactive medical devices used in hospitals, well-designed ones can be highly effective. But poorly designed ones may challenge healthcare practitioners with unexpected behaviour and low predictability. Design based on assumptions and conducted in isolation of user feedback may contribute to medical incident and cause catastrophic consequences. UCD (User-Centred Design) has been in existence for decades, and appears in design and development practice in various forms [1] [2]. It largely relies on end user feedback, together with intuitive design, to ensure the quality of design. It has been endorsed by international standards and regulatory guidances, such as ISO 62366 and NPSA (National Patient Safety Agency) Guidances, and appears to be making an impact across the interactive device industry.

This paper addresses the difficulties for medical devices stakeholders to effectively gather external feedback to apply $\mathrm{UCD}$, and hence suggests potential opportunities for improvement. We analysed established feedback mechanisms, and discuss the feasibility of implementing automatic feedback in medical devices that allows adequate data of clinical use to be gathered from appropriate channels. With the support of such mechanism, medical device manufacturers may apply a set of device-specific analysis algorithms to effectively flag design flaws and inform future design.

\section{Feedback Gathering and Use in Medical Device LIFECYCLE}

MHRA (the UK Medicines and Healthcare products Regulatory Agency) guidelines [3] argues that professional users need to understand manufacturers' intentions in order to be able to use a device effectively and safely. While this is reasonable, we argue that this misses a large part of the picture. For many medical devices there is a gap between theory (known to manufacturers) and practice (known to end users), and often manufacturers design devices without understanding, for example, how their interactive behaviour can increase the likelihood of medical errors during situated use [4].

Theoretically, by gathering and analysing feedback, UCD techniques help to optimise design around the needs, wants and limitations of device users. These methods also allow developers to foresee how users are likely to operate the device and avoid assumptions that may not reflect the context of use. As a result, the design provides better usability and safety. However, current UCD practice in the medical context has not kept up with UCD practitioners' expectations. In fact, an increase in the number and severity of infusion pump problems has been seen over the years. Analysis of the FDA's (Food and Drug Administration) MDRs (Medical Device Report) reveals many device problems that appear to be a result of faulty design: for instance, between 2005-2009, over 56,000 MDRs were associated with the use of infusion pumps. Of these reports, approximately $1 \%$ were reported as deaths, $34 \%$ were reported as serious injuries, and $62 \%$ were reported as malfunctions. Human factors (which include, but are not limited to, use error) are one of the six frequently reported problems. Subsequent root cause analyses revealed that many of these design problems were foreseeable and, therefore, in principle preventable [5].

To understand current UCD practice within the medical device industry and how external feedback is gathered and fed in to the design process, we carried out a series of semistructured interviews in 2010 [4]. Following this study, in 2011, we arranged further meetings and a series of workshops to engage with a larger spectrum of medical device stakeholders to continually explore some of the key issues. Of the 27 
participants: 8 were from global medical device companies; 5 were from design houses or consulting companies; 4 were from small medical device companies; 4 were with a NHS Trust; 4 were regulators, evaluators or from testing labs; and 2 were from research labs.

\section{A. Established Feedback Channels}

Based on the study, we summarise the established feedback channels into two phrases (pre-market and post-market), further explained below.

1) Pre-Market User Engagement: Pre-market user engagement is mainly carried out by the device manufacturer towards fulfilling international standards, such as ISO 60601, ISO 62366, ISO 62304. In the UK, guidance has been provided by the NPSA as part of the"Design for Patient Safety" initiative [7], [8]. This supports UCD by detailing examples of best practice and illustrating device specific issues and recommendations. In America, the FDA also provides guidance and suggested a series of UCD procedures to avoid Use Hazards. Recommendations include: human factors evaluation, summative human factors study, simulated use and clinical evaluation. These resources, to some degree, have promoted and sometimes urged the information flow between medical device end users and manufacturers to assist UCD in the premarket phase.

Our interview participants were all aware of the need for UCD, and have been using various UCD techniques to gather and manage user input and feedback during the design process. For example, some practitioners run focus groups surrounding form factor issues. Scenarios are also a standard technique, and sometimes used in conjunction with focus groups or stakeholder workshops. Other commonly used methods include prototyping, cognitive walkthrough, interviews and usability testing. Some participants were enabled, to some extent, to work with people from other departments. Marketing department was considered to be the major contact point to the user. Thus, collaborative work between design teams and marketing teams was valued.

2) Post-Market Surveillance: In the post-market phase, feedback on safety is given higher priority than feedback on usability. A great deal of feedback on device safety is gathered through post-market safety monitoring and reporting, supported by regulatory agencies.

MDR regulation requires the manufacturer to submit reports to the FDA whenever they are aware of information that reasonably suggests that a device they market may have caused or contributed to a reportable death or serious injury, or has malfunctioned and the malfunction would be likely to cause or contribute to a reportable death or serious injury should it recur.

Similarly, NPSA's NRLS (National Reporting and Learning System) collects confidential reports of patient safety incidents from healthcare staff (across England and Wales) to be analysed by clinicians and safety experts. Based on the analysis, safety alerts and guidance is produced for relevant bodies.
Parallel with NRLS, a formal mechanism is outlined in the MDD (Medical Device Directive) and supported by the MHRA, which logs adverse incidents as they happen with devices. This system is open to reports from healthcare practitioners, patients and medical device manufacturers. Information gathered from reports will go through risk assessment and, where appropriate, investigation undertaken by medical device specialists. Results are distributed in various forms, such as posters, One Liners and Device Bulletins. Stakeholders involved in the report are updated continuously with the progress. The MHRA would be involved in investigating design alternatives if the conclusion revealed serious design flaws.

Less formal mechanisms are deployed by medical device manufacturers involving the use of call centres and training staffs. Participants from international medical device companies spoke of conducting observations in client hospitals. If there is a mismatch between the actual operating pattern and the recommended routine, meetings would be arranged with client's representative to discuss potential solutions, which usually involves re-training or re-configuration. However, small medical device companies might not have the advantage and resources to take such action.

\section{B. Problems with Current Feedback Channels}

Current feedback mechanisms have successfully provoked better design of both medical devices software [9] and hardware [10]. Based on data gathered through our study, an overview of current feedback mechanisms in medical device lifecycle is presented in Fig.1. The lifecycle consists of 13 phases, which are marked on arcs, with the input and output of each phase shown as underlined text. Stakeholders are colourcoded and marked on rings (where EBME stands for ElectroBiomedical Engineering). Dark cells indicate stakeholders involvement in each phase.

Our study reveals that there are a number of problems that are in need of attention. These problems can be summarised by seven groups.

1) Organisational Impact on UCD Practice: Some medical device companies rely on outside consultants, usability engineers or human factor experts, due to the benefits associated with short term contracting. These external personnel are responsible for proposing a design decision clarifying the reasoning behind it. But it has to be accepted by the client. Participants explained that some requests can not be fulfilled because of other limits that the client is facing, stating:

"Sometimes the customer refuses to provide the necessary resources, also due to some related projects, it is very political. We have various problems, most of them are related to the hierarchy of the customer and the decision trees which are rather huge."

When a medical device development process involves subcontracting or outsourcing, getting access to end users could be difficult. Participants explained that sometimes subcontractors might not be allowed access to end users. 


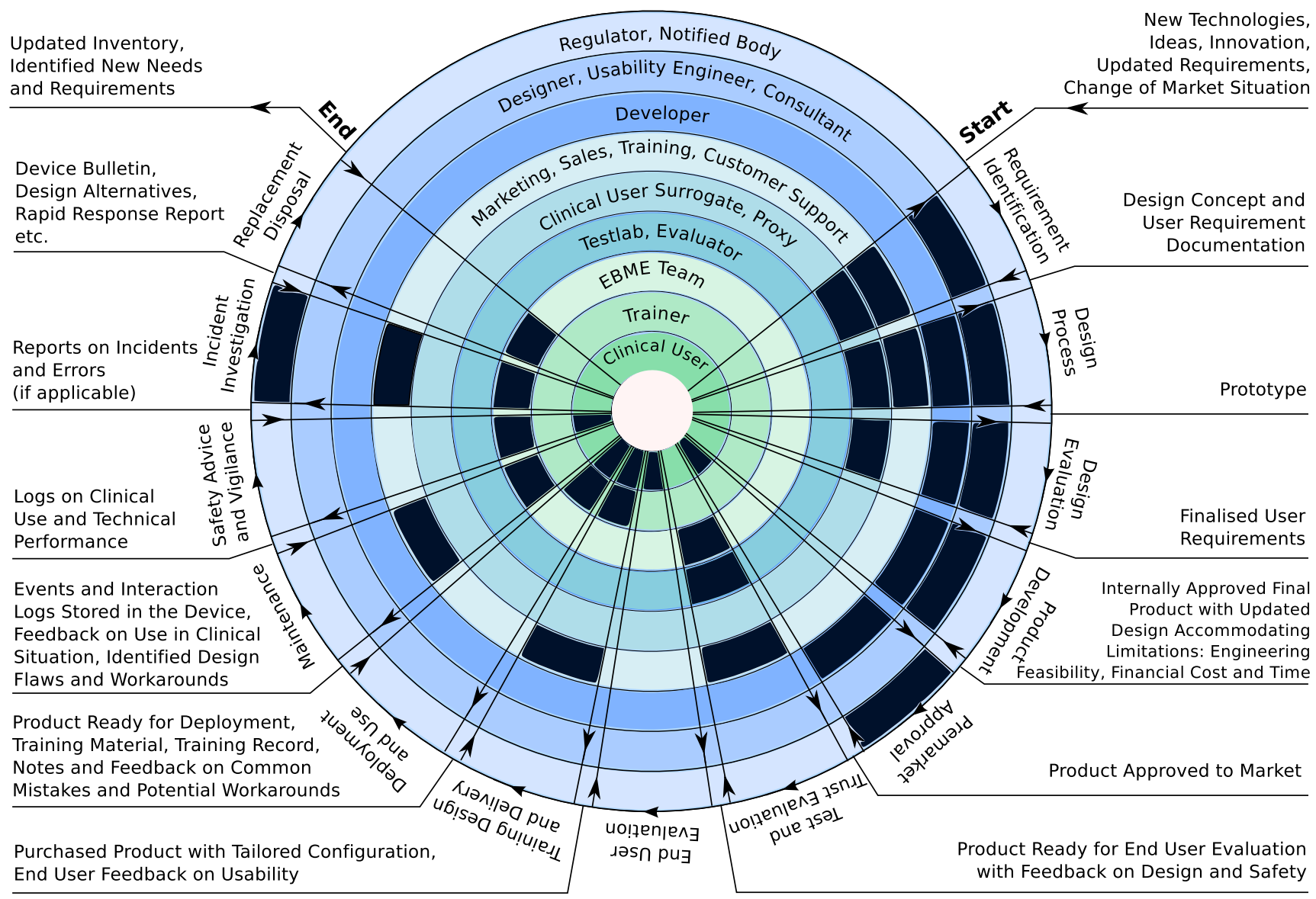

Fig. 1. An Overview of Feedback Mechanisms in Medical Device Lifecycle, Stakeholders Involved and Output Generated

2) Identifying the Right Source of Feedback: Because of the complex nature of medical devices, and the fact that design can involve multiple levels of outsourcing, it is crucial to identify the right group of people to engage with and to prioritise feedback.

Prioritising the competing demands of various stakeholder groups was seen as a challenge and it could be difficult for subcontractors or usability consultant to balance user needs with those of the OEM (Original Equipment Manufacturer). In addition, medical devices can be used by both clinicians and patients. The requirements and feedback coming from these two groups are often quite different.

3) Gaining Access to the Source of Feedback: Clinicians are required to work long hours and with great intensity. A union survey [13] has revealed that $80 \%$ of NHS staff reported an increase workload over the year of 2010, and $77 \%$ increased stress.

Participants expressed their concern about gaining access to end users, stating:

"The user type determines how long you can have the user for, or whether you can get the user at all. When designing medical user interfaces, you can hardly get hold of doctors or nurses, because they are just busy."

It is also reported that small medical device companies struggle to gain access to end users due to low profile and lack of resources. On the other hand, ethical hurdles are considered barriers to all participants from industry regardless of their company size:

"Going back to the ideal ... if you can just go and talk

to people, see what they are really doing in the hospital in the situation, that's what you really want,

but you've got there barriers to go into these

situation, the ethical hurdles and so on."

4) Missing Information on Real Clinical Situation: Traditional UCD techniques can not foresee the complexity of real clinical situation. Though some factors can be controlled for or investigated within a lab, the results from lab based studies don't always generalise to hospital settings due to the difficulty of simulating or replicating a given context.

In 2003, a snapshot audit showed that in two main acute hospitals in a South Wales NHS Trust, there were over 30 different infusion devices in use. Medical device standardisation helped to bring the total number of different infusion devices in use down to 8 [14]. However, infusion devices are only 
one category of interactive medical device out of many that nurses and doctors need to operate in a hospital environment! The familiarity with these devices and their operating routines would have an significant impact on the interaction speed and accuracy:

"The more often the user interacts with the device, the faster they get with it. And some times it's difficult to measure in lab based test where people are unfamiliar with devices, because they interact more slowly with it. If you press the button faster (than it can respond), maybe nothing happens."

Participants spoke of various means to compensate for the deficiency of information on clinical situation. Some device companies have clinical practitioners involved in the design process or on the board, acting as Clinical User Surrogate (See Fig. 1). Though they are not end users, they have an understanding of what the end users will deal with in their working environments. Such approaches are highly feasible both financially and organisationally, but perhaps with limitations.

5) Limitations of Trials and End User Evaluation Before Deployment: Clinical trials and end user evaluation are ideal approaches for feedback gathering, as it involves both real end users and real clinical environment.

As shown in Fig.1, for some medical devices, clinical trial could possibly be the first opportunity for the device to be used in a realistic clinical situation prior to the market. In other words, the trial could be the first stage when a real end user is actually involved. However, participants explained that by the time a product got to clinical trials, sufficient costs had accumulated such that failure was not an option. User tests at this stage would be designed to maximise the chance of getting through the trail. Such trials are structured for regulatory approval, rather than user evaluation and feedback gathering.

In the post-market phase, medical devices may be evaluated by medical device evaluators (e.g., Cedar, SMTL-Surgical Medial devices Test Lab) that report to regulatory bodies (e.g., MHRA). Some NHS Trusts may contract external organisations to conduct test and evaluation, while others are tested and evaluated using the Trust's own EBME team.

Participants reported that though the test and evaluation can generate a large amount of information for feedback, the feedback channel between EBME and designer is rather weak. Fig. 1 shows a clear divide between phases where these two parties are involved in. Though participants spoke of rare cases where good communication between designers and EBME staffs led to a significant improvement in the design of medical devices, due to geographic limitations and organisational impact, sales representatives are often the only contact they can approach, and there is rarely formal acknowledgement from the designer on the reception of such information:

"Very little formal feedback. You might get an

acknowledgement from the rep (representative) or

something like that. But you wouldn't, once the

information is passed on to them, you would not, generally, tend to get anything. You certainly wouldn't get how they would apply, what they've done."
Prior to purchase and deployment, NHS Trusts policy and procedures demand thorough evaluation of devices [15] [16]. Devices that meet procurement criteria can be arranged to come into the Trust for end user evaluation. However, participants spoke of situations where users have been trained on legacy products and may experience difficulties in transferring to new products. If a new product has a similar appearance to the legacy product, though they may function differently, nurses may assume that devices that look alike should behave alike. That can influence the user's perception of new devices, and have an impact on the evaluation result.

The lack of time to gather adequate data from end user evaluation is also raised. As mentioned previously, nurses need to interact with large numbers of devices daily. The total amount of time allocated on a new device during the evaluation period is typically limited.

6) Under-reporting: Participants from regulatory agencies explained that without stakeholders reporting, they wouldn't be aware of an incident, therefore, cannot take further action.

On the other hand, Sari et al [11] evaluated the relative performance of a local routing incident reporting system that feeds into NRLS. They found that though the reviewers were specially trained and inter-rater reliability was good, the implemented routine reporting system missed most patient safety incidents identified by case note review, and detected only $5 \%$ of those incidents that resulted in patient harm.

In addition, relying on users to report all incidents is flawed, as evidence shows that users may not be aware of so-called "unremarkable errors" [12], or understand whether certain incidents (near misses) should be reported, often record limited details about the event and often do not record the identity of the device that was being used at the time.

7) Limitations of Current Logs: When applicable, interactive medical devices record event logs (Fig. 1, Maintenance phase and Safety Advice and Vigilance Phase). Such logs can be used either for routine monitoring or for helping retrospective analysis of known errors. In the majority of cases, these logs are used for maintenance purposes or as a resource in finding out the reason behind a failure in execution or an incident. However, the difficulty of analysing logs is raised.

Currently, logs are processed and presented at a level of abstraction rather than at a level of detailed user interaction, and are rarely used until something fails. In complex software, for which most of current research is based, it has been necessary to do this in order to reduce the amount of data logged. However, this process of abstraction eliminates important information on usability. The manufacturers of devices do not normally provide tools to analyse logs on an usability evaluation level. In some cases, it is reported that not all logs are sequential, which makes exploring the user behavioural pattern extremely challenging.

\section{Enable Feedback in Medical Device Software DESIGN}

Our study reveals the challenges faced by UCD practitioners in incorporating feedback relating to the likely users and 
usage of devices. Post-market feedback is mainly triggered by adverse incidents that have already involved death or injury. And studies have found that user errors are often underreported [17]. On the other hand, traditional UCD techniques cannot effectively help designers foresee the complexity of clinical situations in the premarket phase of the medical device lifecycle. Fig. 1 shows that it's rarely the case when the clinical user and the UCD practitioner are both involved in the same phase, which makes it challenging for both parties to participant in the establishment of an effective feedback channel. However, it also shows that a large amount of clinical usage and usability information is recorded as device $\operatorname{logs}$, which can potentially be used to inform future design.

Logs derived from user interface events have long been viewed as a source for assessing the usability of an interactive system [18]. Hilbert and Redmiles [19] describe an agentbased approach to identify and report back when there is a mismatch between actual usage and usage expectation. However, in general, the majority of modern commercial software and safety critical systems only use event logs as a resource for debugging or investigation. Unfortunately, interactive medical devices have also fallen into this model.

The problem with this model is that it is usually too late to prevent the error. When software crashes, it is not uncommon to be presented with a dialog box that requests the user to send some contextual logging information to the manufacturer about the crash. Similarly, black-box and medical device logs are generally ignored until something bad happens.

Interactive medical device logs should be carefully designed so usability information can be continuously gathered and periodically analysed. Periodical analysis of logs complements Reason's "Swiss Cheese" model [20] where latent errors are not merely blocked but have a higher chance of being discovered, acted upon and fixed.

Though medical device log is just one source of feedback that can be improved and cannot solve all the problems, along with appropriate analysing algorithms, a redesigned and wellintegrated logging system can bridge the gap between designer and developer with more accurate feedback on clinical use.

\section{CONCLUSIONS}

A large range of medical device stakeholders have supported our study by sharing their valuable insights and rich work experiences through interviews, meetings, visits and workshops. The study reveals that current feedback mechanisms in medical device UCD are in need of further tuning. Although the presence of post-marketing surveillance and Corrective And Preventative Action (CAPA) makes developers aware of problems, there is potential for a closer examination of differences between assumed and actual usage.

It is clear that data on device usage should be gathered automatically via improved software design. We have already demonstrated that by applying appropriate analysing algorithms to logs from 58 infusion pumps, the mismatches between design and real clinical practice can be identified [21]. However, since device logs are device specific and not currently standardised, the data to log and corresponding analysing algorithm should be further designed. In addition, automatically gathered data needs to be ready for secondary use. Adequate IT infrastructure is needed to collect, transmit and store the data to centralised databases for further analysis and results communicated to the entire health system.

\section{ACKNOWLEDGMENT}

This work is part of the UK EPSRC funded CHI+MED project (EP/G059063). We thank all our stakeholders for their valuable insight.

\section{REFERENCES}

[1] W. Hudson, User-Centred Survey Results, email posting to CHIWEB@ACM.ORG, 3 May 2000

2] R. Gunther, J. Janis and S. Butler, The UCD Decision Matrix: How, When and Where to Sell User-Centred Design into the Development Cycle, http://www.ovostudios.com/upa2001/, 2001.

[3] Medicines and Healthcare products Regulatory Agency, $D B$ 2006(05):Managing Medical Devices. Device Bulletin, MHRA, London, 2006.

[4] C. Vincent and A. Blandford, Designing for safety and usability: Usercentred techniques in medical device design practice, Presented at meeting of Human Factors and Ergonomics Society, 2011.

[5] Food and Drug Administration, Total Product Life Cycle: Infusion Pump - Premarket Notification: Draft Guidance, 23 April, 2010.

[6] A. Blandford, P. Curzon, H. Thimbleby, et al., CHI-MED multidisciplinary Computer-Human Interaction research for the design and safe use of interactive medical devices, www.chi-med.ac.uk, 2010.

[7] National Patient Safety Agency, Design for patient safety: user testing in the development of medical devices, 1 March, 2010.

[8] National Patient Safety Agency, Design for patient safety: A guide to the design of electronic infusion devices, 24 March, 2010.

[9] National Patient Safety Agency, Rapid Response Report - Safer ambulatory syringe drivers, 16 December 2010.

[10] Medical and Healthcare product Regulatory Agency, Device Bulletin Guidance on the safe and effective use of batteries and chargers for medical devices, October 2005

[11] A. B. Sari, T. A. Sheldon, A. Cracknell, and A. Turnbull, Sensitivity of routine system for reporting patient safety incidents in an NHS hospital: retrospective patient case note review, BMJ, 334(7584):79, January 2007.

[12] D. Furniss, A. Blandford and A. Mayer, Unremarkable Errors: Lowlevel Disturbances in Infusion Pump Use, Proceedings of BCS HCI Conference 2011, Newcastle Upon Tyne, UK, 2011.

[13] S. Ford, Nurses hit by workload hike as cuts force down staff numbers, Nursing Times Net, http://www.nursingtimes.net/nursingpractice/clinical-specialisms/management/nurses-hit-by-workloadhike-as-cuts-force-down-staff-numbers/5021442.article, 8 November, 2010.

[14] P. Lee, A Team Approach to Identify and Manage Risk in Infusion Therapy, British Journal of Nursing, 19(5), pp. 12-18, 2005.

[15] P. Phoenix, NHS Bolton - Medical Devices and Equipment Management Policy, December 2008.

[16] The Newcastle Upon Tyne Hospitals NHS Foundation Trust, Medical Devices Procurement Procedure, March 2008.

[17] A. Blandford, G. Buchanan, P. Curzon, D. Furniss, and H. Thimbleby, Whos looking? Invisible problems with interactive medical devices, Presented at the ACM Conference on Human Factors in Computing System, Atlanta, GA, 2010.

[18] D. M. Hilbert and D. F. Redmiles, Extracting usability information from user interface events, ACM Computing Surveys (CSUR), 32:384-421, December 2000

[19] D. M. Hilbert and D. F. Redmiles, An approach to large-scale collection of application usage data over the Internet, Proceedings of the 20th international conference on Software engineering, pp. 136-145, 1998.

[20] J. T. Reason, Human Error, Cambridge University Press, 1990.

[21] P. Oladimeji, Y. Li, A. Cauchi, P. Eslambolchilar, A. Gimblett, P. Lee, $\mathrm{H}$. Thimbleby, Visualising medical device $\operatorname{logs}$, to be appeared in Proceedings of The first BCS Health in Wales/ehi2 joint Workshop, Wrexham, UK, 8th September, 2011. 